\title{
Raphidioptera et Neuroptera (Insecta, Neuropterida) du Parc national du Mercantour (France)
}

Pierre TILLIER

8 rue d'Aire,

F-95660 Champagne-sur-Oise (France) p.tillier.entomo@free.fr

Publié le 31 décembre 2015

MOTS CLÉS

Neuropterida,

Neuroptera,

Raphidioptera,

liste faunistique,

Mercantour,

France.

KEY WORDS

Neuropterida,

Neuroptera

Raphidioptera,

faunal list

Mercantour,

France.

urn:Isid:zoobank.org:pub:16E615CC-77D6-4EE2-8807-91DF7BF31ED9

Tillier P. 2015. - Raphidioptera et Neuroptera (Insecta, Neuropterida) du Parc national du Mercantour (France), in Daugeron C., Deharveng L., Isaia M., Villemant C. \& Judson M. (eds), Mercantour/Alpi Marittime All Taxa Biodiversity Inventory. Zoosystema 37 (4): 581-594. http://dx.doi.org/10.5252/z2015n4a4

\section{RÉSUMÉ}

Lors de l'Inventaire de la Biodiversité généralisé réalisé dans le Parc national du Mercantour, 36 espèces de Neuropterida, appartenant à trois familles de Neuroptera Linnaeus, 1758 (Coniopterygidae Burmeister, 1839, Hemerobiidae Latreille, 1802, Chrysopidae Schneider, 1851) et une famille de Raphidioptera Martynov, 1938 (Raphidiidae Latreille, 1810), ont été collectées. Trois espèces ont été trouvées pour la première fois en France pendant l'inventaire. Trois autres espèces ont été rarement collectées en France. À partir des résultats de cette étude et de données bibliographiques, une liste faunistique des Neuropterida du Mercantour est proposée.

\section{ABSTRACT}

Raphidioptera and Neuroptera (Insecta, Neuropterida) of the Mercantour National Park (France). During the All Taxa Biodiversity Inventory performed in the Mercantour National Park, 36 species of Neuropterida were collected, belonging to three families of Neuroptera Linnaeus, 1758 (Coniopterygidae Burmeister, 1839, Hemerobiidae Latreille, 1802 and Chrysopidae Schneider, 1851) and one family of Raphidioptera Martynov, 1938 (Raphidiidae Latreille, 1810). Three species were new for France. Three other species had rarely been collected in France. A faunal list of Neuropterida from Mercantour is provided, based on the present study and bibliographic data. 


\section{INTRODUCTION}

L'ordre des Neuropterida est représenté au niveau mondial par 6500 espèces (Aspöck et al. 2001), réparties en trois sousordres: Megaloptera Latreille, 1802, Raphidioptera Martynov, 1938 et Neuroptera Linnaeus, 1758. En France, cet ordre comprend approximativement 185 espèces: trois espèces de Megaloptera (Tillier 2009), 18 espèces de Raphidioptera (Tillier 2012a) et plus de 160 espèces de Neuroptera (Tillier 2013a).

Il $y$ a plus de vingt ans, un premier inventaire de l'entomofaune du Parc national du Mercantour permettait de présenter une liste de cinq Raphidioptera et huit Neuroptera (Cocquempot \& Chambon 1990). Par la suite, les travaux de Leraut (1991a-c; 1992a-c) ont augmenté significativement cette liste et documenté la distribution des Neuropterida présents dans le Mercantour et les régions avoisinantes.

Dans le cadre de l'Inventaire généralisé de la Biodiversité réalisé dans le Parc national du Mercantour (Terrestrial Invertebrates Module of the ATBI Mercantour/Alpi Marittime), plus de 240 spécimens de Neuropterida ont été capturés. Un bilan de ces captures est présenté et une synthèse des connaissances actuelles sur les Neuropterida du Parc national du Mercantour, incluant des données bibliographiques, est exposée.

\section{MATÉRIEL ET MÉTHODES}

Le matériel étudié a été collecté dans le cadre du module «Invertébrés Terrestres» du projet ATBI Mercantour qui a largement contribué à compléter l'inventaire de biodiversité entrepris dans ce parc national (Villemant et al. 2015, ce volume). La région du Mercantour/Alpi Marittime, située au carrefour de plusieurs domaines climatiques et biogéographiques (continental, alpin, méditerranéen et ligurien), représente une grande diversité d'altitudes, d'expositions et de substrats géologiques et pédologiques, source de richesse biologique (Deharveng et al. 2015).

Les collectes d'insectes ont été réalisées entre 2009 et 2011 avec deux pièges malaises standards (noir et blanc) et un piège à interception placés à moins de $100 \mathrm{~m}$ les uns des autres dans chaque site d'échantillonnage. L'emplacement des sites différait d'une année à l'autre, mais dans chaque vallée deux sites étaient toujours choisis, l'un vers $1400-1500 \mathrm{~m}$ d'altitude et l'autre autour de $2000 \mathrm{~m}$. Les sites de collecte étaient localisés dans les communes de Saint-Martin-Vésubie et Saint-Delmas-leSelvage (Alpes-Maritimes) en 2009 (quatre sites), de Saorgue (Alpes-Maritimes) en 2010 (deux sites), Meyronnes et Larche (Alpes-de-Haute-Provence) en 2011 (deux sites) (Fig. 1). Les pièges ont été relevés toutes les deux semaines de mai-juin à septembre-octobre, sur une période qui a varié d'une année à l'autre en fonction des conditions climatiques (chutes de neige).

Tous les échantillons prélevés ont été préservés en alcool et codés. Le codage comprend les indications suivantes: M (Mercantour), 09.11 (années de collecte), BOR, SES, CAÏ, LAR (zones d'étude: Le Boréon, Sestrière, Caïros et Larche), 1400/1500 ou 2000 (altitude en m), M1/M2 (pièges Malaises) ou IN (piège d'interception), T1 à T9 (sessions d'échantillonnage) (Deharveng et al. 2015). Des informations sur les emplacements des pièges et leurs coordonnées géographiques, la durée de l'échantillonnage et la végétation dominante sont données dans le Tableau 1 . Les codes et les dates correspondant aux sessions d'échantillonnage (T1 à T9) sont présentés dans le Tableau 2 .

L'ensemble des spécimens est déposé au Muséum national d'Histoire naturelle, Paris.

\section{RESULTATS}

Au cours des 210 sessions de piégeage, 248 spécimens de Neuropterida (47 Raphidioptera et 201 Neuroptera) ont été capturés.

La liste des espèces est présentée ci-dessous. Pour chaque espèce, outre les synonymes, sont précisés: les références du matériel examiné, la distribution dans le Mercantour avec les noms des localités où l'espèce a été observée.

Les noms d'espèces et les localités entre crochets concernent des espèces qui n'ont été recensées que dans des communes limitrophes du Parc national du Mercantour.

Ordre RAPHIDIOPTERA Martynov, 1938

Famille RAPHIDIIDAE Latreille, 1810

Genre Phaeostigma Navás, 1909

\section{Phaeostigma (Phaeostigma) italogallicum}

(H. Aspöck \& U. Aspöck, 1976)

Raphidia (Phaeostigma) italogallica H. Aspöck \& U. Aspöck, 1976: 65.

CATÉGORIE ZOOGÉOGRAPHIQUE. — Espèce adriato-méditerranéenne stationnaire.

Matériel eXaminé. - M09-SES1400T1-M2 (1 0 ).

Distribution dans le MerCantour. — Saint-Étienne-de-Tinée (Cocquemont \& Chambon 1990); Saint-Dalmas-le-Selvage.

\section{REMARQUE}

Espèce connue de France, d'Italie (Alpes et Apennins) (Aspöck et al. 1991) et du nord-est de l'Espagne (Monserrat \& Papenberg 2010). En France, seules deux stations étaient connues jusqu’alors: Sisteron (Aspöck \& Aspöck 1976) et Saint-Étiennede-Tinée (Cocquemont \& Chambon 1990). La capture d'un mâle à Saint-Dalmas-le-Selvage constitue la troisième mention pour la France (Tillier 2013b). Cette espèce n'est connue que de stations situées entre 500 et $1500 \mathrm{~m}$ d'altitude (Aspöck et al. 1980; Monserrat \& Papenberg 2010).

Genre Dichrostigma Navás, 1909

Dichrostigma flavipes (Stein, 1863)

Raphidia flavipes Stein, 1863: 416.

CATÉGorie ZOOGÉOGRAPHIQUE. - Espèce ponto-méditerranéenne. 


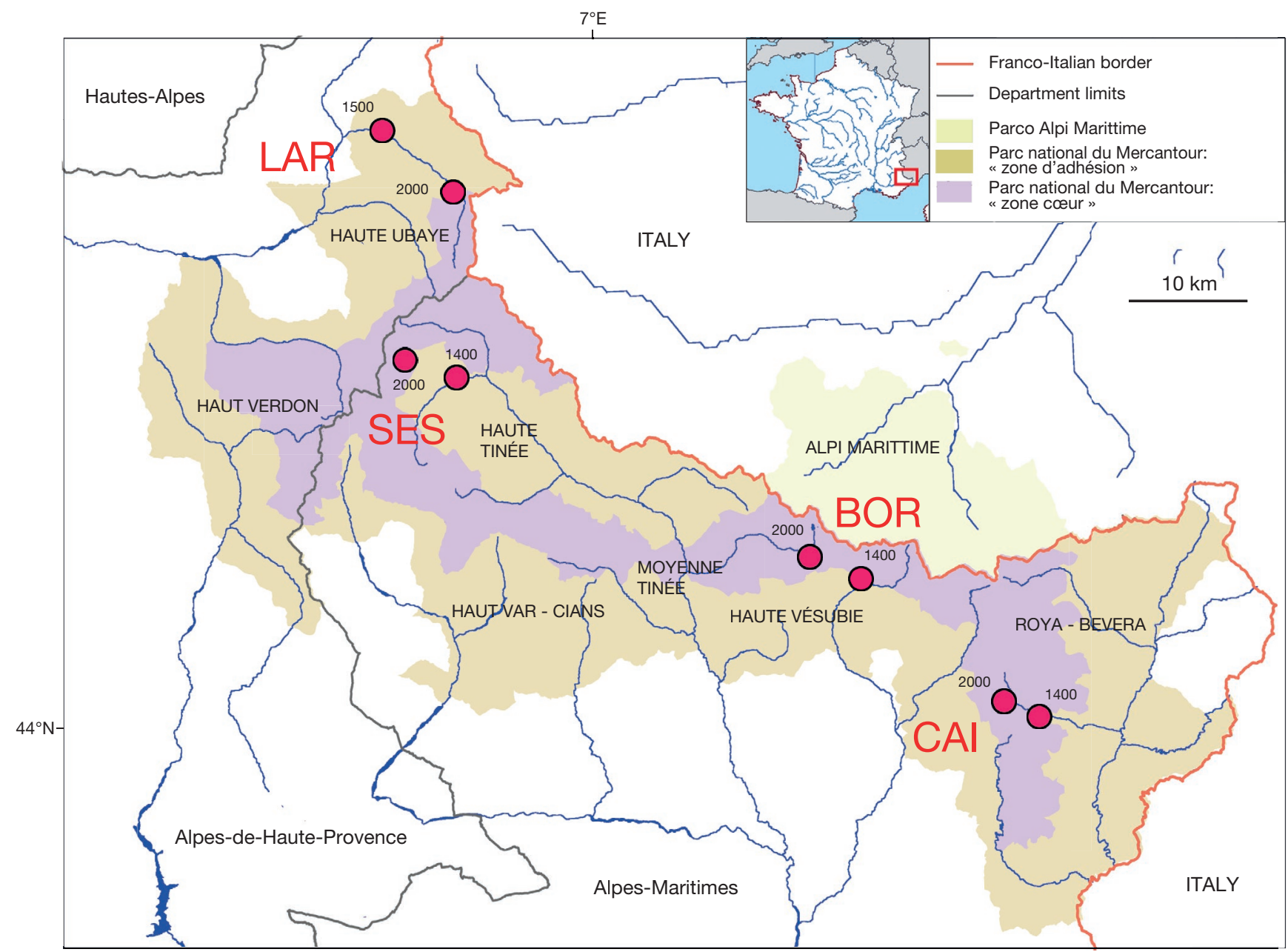

FIG 1. - Localisation des sites de piégeage.

Matériel EXAMINÉ. - M09-SES1400T1-M2 (4 o", 2 \%); M09-SES1400T3-M1 (1 +); M11-LAR1500T1-M1 (4 ơ); M11-LAR1500T1-M2 (2 \%); M11-LAR1500T2-M1 (2 07, 1 \%, 2?); M11-LAR1500T2-M2 (1 \%); M11-LAR1500T3-M1 (1 \%, 1 ?); M11-LAR1500T6-IN2 (1 ơ).

Distribution dans le Mercantour. — Saint-Dalmas-le-Selvage. (Cocquempot \& Chambon 1990); Meyronnes.

Genre Ornatoraphidia H. Aspöck \& U. Aspöck, 1968

\section{Ornatoraphidia flavilabris (Costa, 1855)}

Rhaphidia ophiopsis var. flavilabris Costa, 1855: 4.

CATÉGORIE ZOOGÉOGRAPHIQUE. — Espèce ponto-méditerranéenne.

Matériel eXAMINÉ. - M09-SES1400T1-M2 (2 ơ, 1 \%); M09-SES1400T2-M1 (1 \$); M09-SES1400T3-M2 (1 ơ); M09-SES1400T4-M1 (1 \$).

Distribution dans le Mercantour. — Saint-Étienne-de-Tinée (Cocquempot \& Chambon 1990); Saint-Dalmas-le-Selvage.
Genre Xanthostigma Navás, 1909

\section{Xanthostigma xanthostigma}

(Schummel, 1832)

Raphidia xanthostigma Schummel, 1832: 12.

CATÉgorie ZoogéographiQue. — Espèce sibérienne.

MATÉRIEL EXAMINÉ. — M09-SES2000T2-M1 (2 P); M09-SES2000T3M1 (2 \%); M09-SES2000T4-M1 (1 \%).

Distribution Dans Le Mercantour. — Saint-Dalmas-le-Selvage.

REMARQUE

Espèce nouvelle pour le Mercantour.

Genre Raphidia Linnaeus, 1758

Raphidia (Raphidia) ligurica

Albarda, 1891

Raphidia ligurica Albarda, 1891: 140. 
TABLEAU 1. - Codes et information sur le piégeage : localisation des sites, végétation dominante, période de collecte et nombre de sessions. Départements: (04), Alpes-de-Haute-Provence; (06), Alpes-Martimes.

\begin{tabular}{|c|c|c|c|c|c|c|c|c|c|}
\hline Codes & Année & Commune (*) & Localisation & Végétation & Altitude & Longitude & Latitude & $\begin{array}{l}\text { Période de } \\
\text { collecte }\end{array}$ & $\begin{array}{l}\mathrm{Nb} \text { de } \\
\text { sessions }\end{array}$ \\
\hline $\begin{array}{l}\text { M09-BOR1400- } \\
\text { M1-T1/T8 }\end{array}$ & 2009 & $\begin{array}{l}\text { St-Martin-Vésubie } \\
\text { (06) }\end{array}$ & Le Boréon & $\begin{array}{l}\text { Prairie et forêts } \\
\text { d'épicéa }\end{array}$ & 1540 & 7,2871439 & 44,1146875 & 11.VI-15.X & 8 (T1-T8) \\
\hline $\begin{array}{l}\text { M09-BOR1400- } \\
\text { M2-T1/T8 }\end{array}$ & 2009 & $\begin{array}{l}\text { St-Martin-Vésubie } \\
\text { (06) }\end{array}$ & Le Boréon & $\begin{array}{l}\text { Prairie et forêts } \\
\text { d'épicéa }\end{array}$ & 1549 & 7,2890533 & 44,1143415 & 11.VI-15.X & 8 (T1-T8) \\
\hline $\begin{array}{l}\text { M09-BOR1400- } \\
\text { IN-T1/T8 }\end{array}$ & 2009 & $\begin{array}{l}\text { St-Martin-Vésubie } \\
\text { (06) }\end{array}$ & Le Boréon & $\begin{array}{l}\text { Prairie et forêts } \\
\text { d'épicéa }\end{array}$ & 1540 & 7,2871439 & 44,1146875 & 11.VI-15.X & 8 (T1-T8) \\
\hline $\begin{array}{l}\text { M09-BOR2000- } \\
\text { M1-T1/T8 }\end{array}$ & 2009 & Valdeblore (06) & Col de Salèse & $\begin{array}{l}\text { Prairies, } \\
\text { rhododendrons, } \\
\text { forêt mélèze et } \\
\text { épicéa }\end{array}$ & $\begin{array}{l}2058 \\
t\end{array}$ & 7,23698 & 44,13734 & 10.VI-15.X & 8 (T1-T8) \\
\hline $\begin{array}{l}\text { M09-BOR2000- } \\
\text { M2-T1/T8 }\end{array}$ & 2009 & Valdeblore (06) & Col de Salèse & Forêt de mélèze & 2032 & 7,2352837 & 44,1388598 & 10.VI-15.X & 8 (T1-T8) \\
\hline $\begin{array}{l}\text { M09-BOR2000- } \\
\text { IN-T1/T8 }\end{array}$ & 2009 & Valdeblore (06) & Col de Salèse & Forêt de mélèze & 2047 & 7,2365699 & 44,1372935 & 10.VI-15.X & 8 (T1-T8) \\
\hline $\begin{array}{l}\text { M09-SES1400- } \\
\text { M1-T1/T8 }\end{array}$ & 2009 & $\begin{array}{l}\text { St-Dalmas- } \\
\text { le-Selvage (06) }\end{array}$ & $\begin{array}{l}\text { Vallon de } \\
\text { St-Dalmas : } \\
\text { La Buisse }\end{array}$ & $\begin{array}{l}\text { Prairies, forêt } \\
\text { de mélèze et } \\
\text { feuillus }\end{array}$ & 1437 & 6,8875257 & 44,2848357 & 9.VI-15.X & 8 (T1-T8) \\
\hline $\begin{array}{l}\text { M09-SES1400- } \\
\text { M2-T1/T8 }\end{array}$ & 2009 & $\begin{array}{l}\text { St-Dalmas- } \\
\text { le-Selvage (06) }\end{array}$ & $\begin{array}{l}\text { Vallon de } \\
\text { St-Dalmas : } \\
\text { La Buisse }\end{array}$ & $\begin{array}{l}\text { Prairies, forêt } \\
\text { de mélèze et } \\
\text { feuillus }\end{array}$ & 1421 & 6,8867683 & 44,2850731 & 9.v-15.X & 8 (T1-T8) \\
\hline $\begin{array}{l}\text { M09-SES1400- } \\
\text { IN-T1/T8 }\end{array}$ & 2009 & $\begin{array}{l}\text { St-Dalmas- } \\
\text { le-Selvage (06) }\end{array}$ & $\begin{array}{l}\text { Vallon de } \\
\text { St-Dalmas : } \\
\text { La Buisse }\end{array}$ & $\begin{array}{l}\text { Prairies, forêt } \\
\text { de mélèze et } \\
\text { feuillus }\end{array}$ & 1436 & 6,8876092 & 44,2847834 & 9.VI-15.X & 8 (T1-T8) \\
\hline $\begin{array}{l}\text { M09-SES2000- } \\
\text { M1-T1/T8 }\end{array}$ & 2009 & $\begin{array}{l}\text { St-Dalmas- } \\
\text { le-Selvage (06) }\end{array}$ & Bois de Sestrière & Forêt de mélèze & 1966 & 6,8240421 & 44,2927562 & 8.VI-15.X & 8 (T1-T8) \\
\hline $\begin{array}{l}\text { M09-SES2000- } \\
\text { M2-T1/T8 }\end{array}$ & 2009 & $\begin{array}{l}\text { St-Dalmas- } \\
\text { le-Selvage (06) }\end{array}$ & Bois de Sestrière & Forêt de mélèze & 2011 & 6,8228732 & 44,2925219 & 8.VI-15.X & 8 (T1-T8) \\
\hline $\begin{array}{l}\text { M09-SES2000- } \\
\text { M2-T1/T8 }\end{array}$ & 2009 & $\begin{array}{l}\text { St-Dalmas- } \\
\text { le-Selvage (06) }\end{array}$ & Bois de Sestrière & Forêt de mélèze & 1995 & 6,8235944 & 44,2926706 & 8.VI-15.X & 8 (T1-T8) \\
\hline $\begin{array}{l}\text { M10-CAl1400- } \\
\text { M1-T1/T7 }\end{array}$ & 2010 & Saorge (06) & $\begin{array}{l}\text { Forêt de Caïros : } \\
\text { Vallerasse }\end{array}$ & Forêt de sapins & 1379 & 7,45615 & 44,00338 & 1.VII-15.X & 7 (T1-T7) \\
\hline $\begin{array}{l}\text { M10-CAl1400- } \\
\text { M2-T1/T7 }\end{array}$ & 2010 & Saorge (06) & $\begin{array}{l}\text { Forêt de Caïros : } \\
\text { Vallerasse }\end{array}$ & Forêt de sapins & 1387 & 7,45692 & 44,00343 & 1.VII-15.X & 7 (T1-T7) \\
\hline $\begin{array}{l}\text { M10-CAI1400- } \\
\text { IN-T1/T7 }\end{array}$ & 2010 & Saorge (06) & $\begin{array}{l}\text { Forêt de Caïros : } \\
\text { Vallerasse }\end{array}$ & Forêt de sapins & 1398 & 7,45577 & 44,00344 & 1.VII-15.X & 7 (T1-T7) \\
\hline $\begin{array}{l}\text { M10-CAI2000- } \\
\text { M1-T1/T7 }\end{array}$ & 2010 & Saorge (06) & $\begin{array}{l}\text { Tête de la } \\
\text { Poudrière }\end{array}$ & Forêt de mélèze & 1953 & 7,42407 & 44,01454 & 30.VI-15.X & 7 (T1-T7) \\
\hline $\begin{array}{l}\text { M10-CAI2000- } \\
\text { M2-T1/T7 }\end{array}$ & 2010 & Saorge (06) & $\begin{array}{l}\text { Tête de la } \\
\text { Poudrière }\end{array}$ & Forêt de mélèze & 1992 & 7,42459 & 44,01388 & 30.VI-15.X & 7 (T1-T7) \\
\hline $\begin{array}{l}\text { M10-CAI2000- } \\
\text { IN-T1/T7 }\end{array}$ & 2010 & Saorge (06) & $\begin{array}{l}\text { Tête de la } \\
\text { Poudrière }\end{array}$ & Forêt de mélèze & 1985 & 7,42467 & 44,01400 & 30.VI-15.X & 7 (T1-T7) \\
\hline $\begin{array}{l}\text { M11-LAR1500 } \\
\text { M1 T1-T9 } \\
\text { M11-LAR1500 } \\
\text { M2 T1-T9 } \\
\text { M11-LAR1500 } \\
\text { IN T1-T9 }\end{array}$ & 2011 & Meyronnes (04) & $\begin{array}{l}\text { Rive droite } \\
\text { du ruisseau } \\
\text { Ubayette }\end{array}$ & $\begin{array}{l}\text { Forêt de mélèze } \\
\text { et frêne }\end{array}$ & 1491 & 6,796607 & 44,475409 & 26.V-30.IX & 9 (T1-T9) \\
\hline $\begin{array}{l}\text { M11-LAR2000 } \\
\text { M1 T1-T9 } \\
\text { M11-LAR2000 } \\
\text { M2 T1-T9 } \\
\text { M11-LAR2000 } \\
\text { IN T1-T9 }\end{array}$ & 2011 & Larche(04) & $\begin{array}{l}\text { Ouest du col } \\
\text { de Larche: } \\
\text { forêt de Boisset }\end{array}$ & Forêt de mélèze & 1986 & 6,878456 & 44,422731 & 26.5-30.IX & 9 (T1-T9) \\
\hline
\end{tabular}

TABLEAU 2. - Codes et dates des différentes sessions de piégeage pour chaque site.

\begin{tabular}{|c|c|c|c|c|c|c|c|c|c|}
\hline Site & Année & T1 & T2 & T3 & T4 & T5 & T6 & T7 & T8 \\
\hline BOR 1400 M1, M2, IN & 2009 & 11-24.VI & 24.VI-9.VII & 9-24.VII & 24.VII-13.VIII & 13-27.VIII & 27.VIII-18.IX & 18.IX-5.X & $5-15 . x$ \\
\hline BOR $2000 \mathrm{M1}, \mathrm{M} 2, \mathrm{IN}$ & 2009 & 10-24.VI & 24.VI-9.VII & 9-24.VII & 24.VII-13.VIII & 13-27.VIII & 27.VIII-18.IX & 18.IX-5.X & $5-15 . x$ \\
\hline SES 1400 M1, M2, IN & 2009 & $9-30 . \mathrm{VI}$ & 30.VI-10.VII & 10-23.VII & 23.VII-7.VIII & 7-19.VIII & 19.VIII-22.IX & 22.IX-7.X & $7-15 . X$ \\
\hline SES 2000 M1, M2, IN & 2009 & 8-24.VI & 24.VI-10.VII & 10-23.VII & 23.VII-7.VIII & 7-19.VIII & 19.VIII-22.IX & 22.IX-7.X & $7-15 . X$ \\
\hline CAI 1400 M1, M2, IN & 2010 & 1-16.VII & 16-31.VII & 31.VII-16.VIII & 16-30.VIII & 30.VIII-15.IX & $15-30.1 X$ & $30.1 X-15 . X$ & - \\
\hline CAI 2000 M1, M2, IN & 2010 & 30.VI-16.VII & 16-31.VII & 31.VII-16.VIII & 16-30.VIII & 30.VIII-15.IX & $15-30.1 X$ & 30.IX-15.X & - \\
\hline
\end{tabular}


CATÉGORIE ZOOGÉOGRAPHIQUE. — Espèce adriato-méditerranéenne stationnaire.

MATÉRIEL EXAMINÉ. - M09-SES1400T1-M2 (2 \%); M09-SES2000T2M2 (1 Ơ); M09-SES2000T2-IN (1 \%); M11-LAR2000T2-M2 (1 + ).

Distribution dans le Mercantour. - Saint-Dalmas-le-Selvage (Cocquemont \& Chambon 1990); Larche.

\section{REMARQUE}

Cette espèce adriato-méditerranéenne, rare et très localisée (Aspöck et al. 1991), est connue de moins de dix stations européennes (France, Italie, Suisse - Aspöck \& Aspöck 1966; Aspöck et al. 1974a, b; Cocquemont \& Chambon 1990; Güsten 1998; Rausch et al. 2004; Haring et al. 2011). Pour la France, seules deux stations étaient connues jusqu'alors, toutes deux sur la commune de Saint-Dalmas-le-Selvage (Cocquemont \& Chambon 1990). Quatre des cinq spécimens capturés au cours de cette étude proviennent de cette même commune et le cinquième de la commune de Larche. En France, cette espèce semble ainsi très localisée, puisqu' elle n'est connue que de quelques stations d'altitude du Mercantour (Tillier 2013b).

Genre Puncha Navás, 1915

Puncha ratzeburgi (Brauer, 1876)

Raphidia ratzeburgi Brauer, 1876: 277.

Catégorie ZOOGÉOGRAPHIQUe. — Espèce centro-européenne.

Matériel examiné. - M09-SES1400T2-M1 (1 O o ); M09-BOR1400T2-M2 (1 ○", 1 \%); M09-BOR1400T3-M2 (1 ㅇ) ; M09-SES1400T4-M2 (1 ㅇ); M09-SES2000T2-M2 (2 ơ); M10-CAÏ2000T1-M2 (1 ơ).

Distribution dans le Mercantour. - Saint-Étienne-de-Tinée (Cocquempot \& Chambon 1990); Saint-Martin-Vésubie, SaintDalmas-le-Selvage, Saorge.

Ordre NEUROPTERA

Famille ChrYSOPIDAE Schneider, 1851

Genre Nineta Navás, 1912

Nineta flava (Scopoli, 1763)

Hemerobius flavus Scopoli, 1763: 270.

CATÉGORIE ZOOGÉOGRAPHIQUE. — Espèce centro-européenne.

Distribution danS LE MERCANTOUR. — [Barcelonnette], Entraunes, Villard d'Abas (Leraut 1992c; Canard et al. 1998).

Nineta pallida (Schneider, 1846)

Chrysopa pallida Schneider, 1846: 99

Catégorie zoogéographiQue. — Espèce centro-européenne.

Distribution DANS le MERCANTOUR. — Entraunes (Leraut 1992c; Canard et al. 1998).
Genre Chrysopa Leach in Brewster, 1815

Chrysopa perla (Linnaeus, 1758)

Hemerobius perla Linnaeus, 1758: 549.

Chrysopa reticulata Curtis, 1834: 520.

CATÉgorie ZoogéographiQue. - Espèce sibérienne.

Matériel EXAMINÉ. - M09-BOR1400T1-M2 (1 o", 1 \%); M09-SES1400T1-M2 (1 ơ, 1 \%); M09-SES1400T2-M2 (1 ㅇ); M09-SES2000T3-M1 (1 ९); M11-LAR1500T1-M2 (2 ơ).

Distribution dans le Mercantour. — Saint-Dalmas-le-Selvage, Saint-Étienne-de-Tinée (Cocquempot \& Chambon 1990) ; Jausiers (Leraut 1992c); Saint-Martin-Vésubie, Meyronnes.

\section{Chrysopa abbreviata Curtis, 1834}

Chrysopa abbreviata Curtis, 1834: 520.

CATÉgorie ZOOGÉOGRAPHIQUe. — Espèce sibérienne.

Distribution DANS LE MERCANTOUR. — Uvernet (Leraut 1992c).

Chrysopa gibeauxi (Leraut, 1989)

Metachrysopa pallens gibeauxi Leraut, 1989: 106.

CATÉgorie ZOOGÉOGRAPHIQUe. — Espèce boréo-alpine?

MatéRIEL EXAMINÉ. — M09-BOR1400T5-IN (1 ?).

Distribution Dans le MERCANTOUR. — [Barcelonette] (Leraut 1992b); Saint-Martin-Vésubie.

\section{REMARQue}

Ce taxon a été mis en synonymie avec Chrysopa pallens (Rambur, 1838) par Aspöck et al. (2001), mais l'étude des genitalia de spécimens capturés récemment en France et en Pologne a permis de démontrer qu'il s'agit d'une bona species (Tillier et al. 2014). En France, c'est une chrysope d'altitude, connue uniquement des Hautes-Alpes, des Alpes-de-HauteProvence et de Haute-Loire (Leraut 1989, 1992b; Canard et al. 2011) et dont les données de collecte sont rares. Un spécimen a été capturé pour la première fois dans le Parc du Mercantour.

Genre Pseudomallada Tsukaguchi, 1995

[Pseudomallada flavifrons (Brauer, 1851)]

Chrysopa flavifrons Brauer, 1851: 6.

CATÉgorie ZOOGÉOgRAPHIQUe. — Espèce holoméditerranéenne expansive.

Distribution dans Le MerCANTOUR. — [Barcelonnette] (Leraut 1992c). 


\section{Pseudomallada prasina (Burmeister, 1839)}

Dichochrysa prasina Burmeister, 1839: 981.

CATÉGORIE ZOOGÉOGRAPHIQUE. - Espèce polycentrique expansive, sibérienne et méditerranéenne.

Matériel examiné. - M09-SES1400T3-M2 (1 9); M11-LAR1500T7-M2 (1 ); M11-LAR1500T3-M2 (1 ○’).

Distribution dans le MerCantour. - Commune dans les AlpesMaritimes et de Haute-Provence (Leraut 1992c); Saint-Dalmas-leSelvage, Meyronnes.

\section{Pseudomallada ventralis (Curtis, 1834)}

Chrysopa ventralis Curtis, 1834: 520.

CATÉGORIE ZOOGÉOGRAPHIQUE. - Espèce centro-européenne.

Matériel examiné. - M09-SES1400T4-M2 (1 \&); M11-LAR1500T3-M2 (1 \%).

Distribution DANS LE MERCANTOUR. - [Barcelonnette] (Leraut 1992c); Saint-Dalmas-le-Selvage, Meyronnes.

\section{Genre Cunctochrysa Hölzel, 1970 \\ Cunctochrysa albolineata Killington, 1935}

Cunctochrysa albolineata Killington, 1935: 87.

CATÉGOrIE ZOOGÉOGRAPHIQUE. — Espèce sibérienne.

MatéRIEL EXAMINÉ. - M09-BOR2000T3-IN (1 ơ).

Distribution DANS LE MERCANTOUR. - Jausiers, Uvernet-Fours (Leraut 1992c); Valdebore.

\section{[Cunctochrysa bellifontensis Leraut, 1988]}

Cunctochrysa bellifontensis Leraut, 1988: 230.

CATÉGORIE ZOOGÉOGRAPHIQUE. - Incertain à cause des doutes concernant son statut (voir Remarque).

Distribution dans le Mercantour. - [Barcelonnette, La Condamine] (Leraut 1992c).

\section{ReMARQue}

Ce taxon a été mis en synonymie avec Cunctochrysa albolineata (Killington, 1935) par Aspöck et al. (2001), mais nous ne les suivons pas ici, estimant que son statut et sa répartition restent à préciser.

Genre Peyerimhoffina Lacroix, 1920

Peyerimhoffina gracilis (Schneider, 1851)

Chrysopa gracilis Schneider, 1851: 72.
CATÉGORIE ZOOGÉOGRAPHIQUE. - Espèce polycentrique, centroeuropéenne et méditerranéenne.

MATÉRIEL EXAMiné. - M09-BOR1400T6-M1 (2 ơ).

Distribution dans le MerCantour. — Pra-Loup (Leraut 1992c); Saint-Martin-Vésubie.

Genre Chrysoperla Steinmann, 1964

Chrysoperla lucasina (Lacroix, 1912)

Chrysopa lucasina Lacroix, 1912: 203.

CATÉgorie zoOgÉographiQue. — Espèce holoméditerranéenne expansive.

Matériel eXAMiné. - M09-BOR1400T1-M2 (1 ơ, 1 9); M09-BOR1400T1-M1 (2 O", 1 \%); M09-BOR2000T1-M1 (1 ○’, 2 ㅇ); M09-BOR2000T2-M1 (1 ㅇ) ; M09-SES1400T1-M1 (2 $\left.0^{7}\right)$; M09-SES1400T1-M2 (3 ơ); M09-SES2000T1-M1 (3 ᄋ); M09-SES2000T1-M2 (4 ơ, 1 ९); M09-SES2000T1-IN (1 0 , 1 \%).

Distribution dans Le MerCANTOUR. - Col de la Cayolle (Leraut 1992c) ; Saint-Martin-Vésubie, Valdebore, Saint-Dalmas-le-Selvage.

REMARQUE

Très commune sur feuillus, moins sur résineux, jusqu'à $2000 \mathrm{~m}$ localement (Leraut 1992c).

\section{Chrysoperla sp., groupe carnea}

MATÉRIEL EXAMINÉ. - M09-BOR1400T1-IN (1 ?); M09-SES1400T6M2 (1 ơ); M09-SES1400T3-IN (1 \&).

Distribution dans le MerCantour. - Cocquempot \& Chambon 1990; Leraut 1992c; Saint-Martin-Vésubie, Saint-Dalmas-le-Selvage.

REMARQUE

La taxonomie des espèces du groupe carnea s.l. (Stephens, 1836) étant confuse et leur identification sur la base de critères morphologiques sujette à caution (à l'exception de lucasina Lacroix, 1912, mediterranea [Hölzel, 1972] et renoni [Lacroix, 1933]), les trois exemplaires capturés n’ont pas été identifiés. Pour ces mêmes raisons, les données bibliographiques sont regroupées ici.

Famille HeMEROBIIDAE Latreille, 1802

Genre Hemerobius Linnaeus, 1758

Hemerobius (Hemerobius) humulinus Linnaeus, 1758

Hemerobius humulinus Linnaeus, 1758: 550.

Catégorie zoOgÉographique. - Espèce holarctique.

MATÉRIEL EXAMINÉ. - M10-CAÏ1400T2-M2 (1 \&).

Distribution dans le Mercantour. — Fours (Leraut 1992c); Saorge. 


\section{Hemerobius (Hemerobius) simulans Walker, 1853}

Hemerobius simulans Walker, 1853: 285.

CATÉGORIE ZOOGÉOGRAPHIQUE. — Espèce holarctique.

Distribution Dans le MerCantour. — Pra-Loup (Leraut 1992c).

\section{Hemerobius (Hemerobius) stigma \\ Stephens, 1836}

Hemerobius stigma Stephens, 1836: 112.

CATÉgorie ZOOgÉographique. — Espèce holarctique.

Distribution dans le Mercantour. — Col de la Cayolle (Leraut 1992c).

\section{Hemerobius (Hemerobius) ballaudi Leraut, 1991}

Hemerobius ballaudi Leraut, 1991: 189.

CATÉGORIE ZOOGÉOGRAPHIQUE. - Espèce décrite et citée uniquement des Pyrénées et des Alpes françaises.

Distribution dans le MerCantour. - Col de la Cayolle (Leraut $1991 b$; 1992c)

\section{REMARQUE}

Ce taxon a été mis en synonymie avec Hemerobius (Hemerobius) stigma Stephens, 1836 par Aspöck et al. (2001), mais nous ne les suivons pas ici, estimant que son statut et sa répartition restent à préciser.

\section{Hemerobius (Hemerobius) pini}

Stephens, 1836

Hemerobius pini Stephens, 1836: 111.

CATÉGORIE ZOOGÉOGRAPHIQUE. — Espèce sibérienne.

Matériel examiné. - M09-BOR1400T1-M1 (1 O'); M09-BOR1400T1-M2 (2 \%).

Distribution Dans le MerCantour. — Pra-Loup (Leraut 1992c); Saint-Martin-Vésubie.

\section{Hemerobius (Hemerobius) contumax Tjeder, 1932}

Hemerobius contumax Tjeder, 1932: 195.

CATÉGORIE ZOOGÉOGRAPHIQUE. - Espèce polycentrique, centroeuropéenne et méditerranéenne.

Matériel examiné. - M09-BOR1400T1-M2 (1 9); M10-CAÏ1400T6-IN (1 ?).

Distribution dans le Mercantour. - [Tournoux (Leraut 1992c)]; Saint-Martin-Vésubie, Saorge.

\section{Hemerobius (Hemerobius) atrifrons \\ McLachlan, 1868}

Hemerobius atrifrons McLachlan, 1868: 184.

CATÉGORIE ZOOGÉOGRAPHIQUE. - Espèce sibérienne.

Matériel eXAMiné. - M09-SES1400T2-M2 (1 9); M09-BOR2000T3-M1 (2 ơ).

Distribution DANS Le MerCantour. - Col de la Cayolle (Leraut 1992c), Saint-Martin-Vésubie, Saint-Dalmas-le-Selvage.

\section{Hemerobius (Hemerobius) nitidulus}

Fabricius, 1777

Hemerobius nitidulus Fabricius, 1777: 244.

Catégorie zoogéographique. - Espèce sibérienne.

Distribution dans le MerCantour. — Col de la Cayolle (Leraut 1992c).

\section{Hemerobius (Hemerobius) australis Leraut, 1992}

Hemerobius australis Leraut, 1992: 9.

CATÉGORIE ZOOGÉOGRAPHIQUE. - Espèce décrite et citée uniquement des Alpes françaises et des Pyrénées.

Distribution dans le Mercantour. - Col de la Cayolle, [Enchastrayes], Jausiers, Pra-Loup (Leraut 1992a, c).

REMARQUE

Ce taxon a été mis en synonymie avec Hemerobius (Hemerobius) nitidulus Fabricius, 1777 par Aspöck et al. (2001), mais nous ne les suivons pas ici, estimant que son statut et sa répartition restent à préciser.

\section{Hemerobius (Hemerobius) schedli Hölzel, 1970}

Hemerobius schedli Hölzel, 1970: 104.

Matériel eXAminé. - M09-BOR2000T2-M1 (1 \%); M09-BOR2000T3-M2 (1 ㅇ); M09-BOR2000T4-M1 (2 ơ).

CATÉgorie ZOOgÉOgRAPHiQUe. — Espèce centro-européenne.

Distribution dans le Mercantour. — Valdebore.

REMARQUE

Cette espèce dont la répartition est limitée aux grands massifs montagneux est assez peu capturée en Europe. Une seule station française, située dans les Pyrénées, était jusqu’alors connue (Aspöck et al. 1980). La capture de quatre spécimens dans une station sur la commune de Valdeblore, constitue ainsi la deuxième localité française de l'espèce en l'état actuel des connaissances (Tillier 2012c). 


\author{
Hemerobius (Hemerobius) micans \\ Olivier, 1792
}

Hemerobius micans Olivier, 1792: 63.

CATÉGORIE zOOGÉOGRAPHIQUE. - Espèce polycentrique, centroeuropéenne et méditerranénne.

Distribution dans le Mercantour. — Col de la Cayolle (Leraut 1991a; Leraut 1992c).

\section{Hemerobius (Hemerobius) lutescens \\ Fabricius, 1793}

Hemerobius lutescens Fabricius, 1793: 84.

CATÉgorie Zoogéographique. — Espèce sibérienne.

Distribution dans le Mercantour. - Col de la Cayolle (Leraut 1992c).

\section{[Hemerobius (Hemerobius) gilvus}

Stein, 1863]

Hemerobius gilvus Stein, 1863: 418.

CATÉGORIE ZOOGÉOGRAPHIQUE. — Espèce holoméditerranéenne expansive.

Distribution DANS LE MERCANTOUR. — [Maurin] (Leraut 1992c).

Genre Wesmaelius Krüger, 1922

\section{Wesmaelius (Wesmaelius) quadrifasciatus}

(Reuter, 1894)

Hemerobius concinnus var. quadrifasciatus Reuter, 1894: 12

CATÉgorie ZOOGÉOGRAPHIQUE. — Espèce sibérienne.

MATÉRIEL EXAMINÉ. —M09-SES2000T2-M1 (1 \%); M09-SES1400T1IN (1 O); M09-SES2000T1-IN (1 o" + 1 ?); M09-SES2000T3M1 (1 ơ); M09-SES2000T4-M1 (1 क); M11-LAR1500T1-M1 (1 \%); M11-LAR2000T4-IN2 (2 \%); M11-LAR2000T4-M2 (3 +); M11-LAR2000T5-IN1 (1 \%); M11-LAR2000T5-M2 (1 ).

Distribution dans Le MerCantour. - Col de la Cayolle (Leraut 1992c); Saint-Dalmas-le-Selvage, Larche.

\section{Wesmaelius (Kimminsia) fassnidgei}

(Killington, 1933)

Boriomyia fassnidgei Killington, 1933: 57.

Catégorie ZoogéographiQue. — Espèce centro-européenne.

Matériel examiné. - M09-BOR2000T3-M2 (2 ơ, 2 \%) ; M09-BOR2000T4-M2 (2 ᄋ); M09-SES2000T4-IN (1 ).

Distribution dans le Mercantour. — Col de la Cayolle (Leraut 1992c); Valdebore, Saint-Dalmas-le-Selvage.

\section{Wesmaelius (Kimminsia) nervosus}

(Fabricius, 1793)

Hemerobius nervosus Fabricius, 1793: 85.

Kimminsia betulina Strøm, 1788: 387.

Catégorie zoOgÉographique. — Espèce holarctique.

Matériel eXAminé. - M09-BOR2000T2-M2 (1 o')

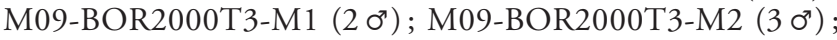
M09-BOR2000T4-M2 (1 ơ + 2 \%); M09-SES2000T4-M1 (1 ᄋ); M10-CAÏ2000T1-M2 (1 \%).

Distribution dans le MerCantour. — Saint-Dalmas-le-Selvage (Cocquempot \& Chambon 1990); Valdebore, Saorge.

\section{Wesmaelius (Kimminsia) malladai}

(Navás, 1925)

Hemerobius malladai Navás, 1925: 30.

Kimminsia killingtoni Morton in Fraser, 1942: 80

CATÉgorie ZoogéographiQue. — Espèce arctico-alpine.

Matériel EXAMiné. - M10-CAÏ2000T1-M2 (1 9 ); M11-LAR2000T4-M2 (1 ơ); M11-LAR2000T2-M1 (1 ).

Distribution dans Le MerCantour. - Saint-Dalmas-le-Selvage (Cocquempot \& Chambon 1990); col de la Cazolle (Leraut 1992c); Saorge, Larche.

\section{Wesmaelius (Kimminsia) subnebulosus} (Stephens, 1836)

Hemerobius subnebulosus Stephens, 1836: 107.

CATÉGORIE ZOOGÉOGRAPHIQUe. — Espèce holoméditerranéenne expansive.

Matériel examiné. - M09-BOR1400T1-M1 (1 \&); M09-BOR2000T1-M1 (1 \&); M10-CAÏ2000T1-M2 (1 ơ, 2 \%); M10-CAII2000T2-M2 (1 O').

Distribution dans le Mercantour. — Col de la Cayolle (Leraut 1992c). Saint-Martin-Vésubie, Valdebore, Saorge.

Genre Sympherobius Banks, 1904

\section{Sympherobius (Sympherobius) elegans}

(Stephens, 1836)

Hemerobius elegans Stephens, 1836: 113.

Sympherobius striatellus Morton, 1914: 210.

CATÉgorie zoOgÉographique. — Espèce holoméditerranéenne expansive.

Matériel EXAMiné. - M11-LAR1500T4-IN1 (1 \&); M11-LAR1500T2-M2 (1 0 ).

Distribution dans le Mercantour. — Meyronnes. 
REMARQUE

Espèce nouvelle pour le Mercantour.

Sympherobius (Niremberge) fuscescens

(Wallengren, 1863)

Hemerobius fuscescens Wallengren, 1863: 22.

Sympherobius inconspicuus Banks, 1906: 29.

Catégorie zoogéographiQue. — Espèce sibérienne.

MATÉRIEl EXAMinÉ. - M09-BOR1400T2-M2 (1 9).

Distribution DANS LE Mercantour. - [Barcelonette] (Leraut 1992c); Saint-Martin-Vésubie.

\section{REMARQUE}

Espèce signalée pour la première fois du Parc du Mercantour.

\section{Sympherobius (Niremberge) pellucidus (Walker, 1853)}

Hemerobius pellucidus Walker, 1853: 284.

CATÉGORIE ZOOGÉOGRAPHIQUE. — Espèce holoméditerranéenne expansive.

Matériel eXaminé. - M09-BOR1400T5-M2 (2 \&); M09-SES2000T1-IN (1 \%)

Distribution dans le Mercantour. — Pra-Loup (Leraut 1992c); Saint-Martin-Vésubie, Saint-Dalmas-le-Selvage.

Genre Megalomus Rambur, 1842

Megalomus tortricoides Rambur, 1842

Megalomus tortricoides Rambur, 1842: 604.

CATÉGORIE ZOOGÉOGRAPHIQUE. - Espèce holoméditerranéenne expansive.

Distribution dans Le MerCantour. — Saint-Dalmas-le-Selvage, Saint-Étienne-de-Tinée (Cocquempot \& Chambon 1990).

Megalomus hirtus (Linnaeus, 1761)

Hemerobius hirtus Linnaeus, 1761: 382.

CATÉGORIE ZOOGÉOGRAPHIQUE. - Espèce polycentrique, centroeuropéenne et méditerranéenne.

Matériel eXAminé. — M10-CAÏ1400T1-M2 (1 ơ).

Distribution dans le Mercantour. — Saorge.

REMARQUE

Espèce nouvelle pour le Mercantour.
Genre Drepanepteryx Leach in Brewster, 1815

Drepanepteryx algida

(Erichson in Middendorff, 1851)

(Fig. 2)

Hemerobius algidus Erichson in Middendorff, 1851: 68.

Catégorie zoogéographiQue. - Espèce sibérienne.

Matériel examiné. - M09-BOR2000T7-M1 (1 o ); M09-SES1400T6-M1 (2 ơ, 1 ९); M09-SES1400T6-M2 (1 o", 1 \%); M09-SES2000T5-M2 (1 \%); M09-SES2000T6-M2 (3 ه", 1 \%); M10-CAÏ2000T4-M1 (1 o ); M10-CAÏ2000T5-M2 (1 ơ); M10-CAÏ2000T5-M1 (2 ơ); M10-CAÏ2000T6-M1 (3 ơ, 3 \%); M10-CAÏ2000T7-M1 (2 ơ, 1 †); M10-CAÏ2000T7-M2 (2 \%); M11-LAR2000T7-M1 (1 +); M11-LAR1500T8-M1 (1 ơ); M11-LAR1500T9-M1 (2 $\%$ ).

Distribution DANS LE MERCANTOUR. — Valdebore, Saint-Dalmasle-Selvage, Saorge, Meyronnes, Larche.

REMARQUE

Espèce à large répartition, depuis les Alpes jusqu'en ExtrêmeOrient. En Europe de l'Ouest, D. algida est connue de Suisse, dans les cantons du Valais et de Genève (Tjeder 1963), et en Italie, dans le Val d'Aoste (Ruffo \& Stoch 2006), localités proches des frontières françaises. La présence de l'espèce dans les Alpes françaises était donc supposée, mais aucune capture ne venait l'attester. Au cours de cet inventaire, plusieurs spécimens ont été capturés dans neuf stations différentes. L'espèce semble ainsi assez commune dans la zone d'étude. Ces captures dans le Mercantour constituent les premières pour la France (Tillier 2012b).

Genre Micromus Rambur, 1842

Micromus variegatus (Fabricius, 1793)

Hemerobius variegatus Fabricius, 1793: 85.

Catégorie zoogéographique. - Espèce sibérienne.

Matériel eXAminé. - M10-CAÏ1400T4-M2 (1 ơ).

Distribution dans le Mercantour. - Saorge.

REMARQUE

Espèce nouvelle pour le Mercantour.

Micromus paganus (Linnaeus, 1767)

Hemerobius paganus Linnaeus, 1767: 912.

Micromus lineosus Rambur, 1842: 416.

CATÉgorie zoogéographiQue. — Espèce sibérienne.

Matériel examiné. - M09-BOR1400T5-M2 (1 ơ ); M10-CAÏ2000T1-M2 (2 o"); M10-CAÏ1400T3-M1 (1 o); M11-LAR1500T1-M2 (1 ơ); M11-LAR2000T4-M1 (1 \%); M11-LAR1500T7-M2 (1 + ). 
Distribution dans le Mercantour. — Saint-Dalmas-le-Selvage (Cocquempot \& Chambon 1990); Saint-Martin-Vésubie, Saorge, Meyronnes, Larche.

\section{Micromus lanosus (Zeleny, 1962)}

Stenomicromus lanosus Zeleny, 1962: 246.

Catégorie zoOgéographiQue. — Espèce centro-européenne.

Distribution dans le Mercantour. — Villars d'Abas (Leraut 1991c, 1992c).

Famille CONIOPTERYGIDAE Burmeister, 1839

Genre Helicoconis Enderlein, 1905

Helicoconis (Helicoconis) hirtinervis Tjeder, 1960

Helicoconis hirtinervis Tjeder, 1960: 313.

CATÉgorie ZOOgÉographique. — Espèce centro-européenne.

Matériel examiné. - M09-BOR1400T2-M1 (1 0 o ); M10-CAÏ2000T1-M2 (2 o ); M10-CAÏ2000T2-M2 (2 ơ); M11-LAR2000T2-M1 (2 ơ).

Distribution dans le Mercantour. - Saint-Martin-Vésubie, Saorge, Larche (Tillier 2013c).

\section{REMARQUE}

Cinq spécimens ont été capturés pour la première fois en France dans trois communes du Parc national du Mercantour. Cette espèce est connue d'Allemagne, du Danemark, d'Espagne, d'Italie, de Slovénie et de Suisse (Aspöck et al. 2001). Sa présence en France s'inscrit donc logiquement dans une répartition ouest-européenne (Tillier 2013c).

\section{Helicoconis (Fontenellea) hispanica} Ohm, 1965

Helicoconis hispanica Ohm, 1965: 196.

CATÉGORIE ZOOGÉOGRAPHIQUE. — Espèce atlanto-méditerranéenne.

MatéRIEl EXAMINÉ. - M10-CAÏ2000T2-M2 (1 ơ).

Distribution dans le Mercantour. — Saorge (Tillier 2013c)

\section{REMARQUE}

Espèce connue du Maroc, d'Espagne, de Sicile et de Sardaigne (Aspöck et al. 2001). La capture d'un mâle sur la commune de Saorge constitue la première mention de cette espèce en France (Tillier 2013c).
Genre Coniopteryx Curtis, 1834

\section{Coniopteryx (Coniopteryx) pygmea} Enderlein, 1906

Coniopteryx pygmea Enderlein, 1906: 201.

CATÉGORIE ZOOGÉOGRAPHIQUE. — Espèce polycentrique, méditerranéenne et sibérienne.

Matériel examiné. - M09-BOR2000T3-M2 (1 O');

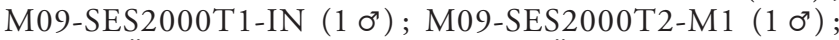
M10-CAÏ2000T1-M1 (2 o'); M10-CAÏ2000T1-M2 (1 ơ ); M10-CAÏ1400T2-M2 (1 ơ); M10-CAÏ1400T4-M2 (4 o ); M10-CAÏ1400T5-M2 (2 0').

Distribution dans le Mercantour. — Saint-Martin-Vésubie, Saint-Dalmas-le-Selvage, Saorge.

REMARQUE

Espèce nouvelle pour le Mercantour.

\section{Coniopteryx (Coniopteryx) tineiformis Curtis, 1834}

Coniopteryx tineiformis Curtis, 1834: 528.

CATÉgorie zoOgÉographique. - Espèce holarctique.

Matériel examiné. - M09-SES1400T3-M2 (1 ơ ); M11-LAR1500T1-M2 (1 ơ).

Distribution DANS Le Mercantour. — Saint-Dalmas-le-Selvage, Meyronnes.

REMARQUE

Espèce nouvelle pour le Mercantour.

Genre Parasemidalis Enderlein, 1905

Parasemidalis fuscipennis (Reuter, 1894)

Coniopteryx fuscipennis Reuter, 1894: 32.

Parasemidalis annae Enderlein, 1905: 198.

CATÉgorie zoOgÉographique. - Espèce holarctique.

Matériel examiné. - M10-CAÏ2000T2-M1 (1 \%); M10-CAÏ2000T2-IN (1 \&); M11-LAR1500T2-M1 (1 \&).

Distribution dans le Mercantour. — Saorge, Meyronnes.

REMARQUE

Espèce nouvelle pour le Mercantour.

Genre Conwentzia Enderlein, 1905

Conwentzia psociformis (Curtis, 1834)

Coniopteryx psociformis Curtis, 1834: 528.

Conwentzia pineticola Enderlein, 1905: 10. 


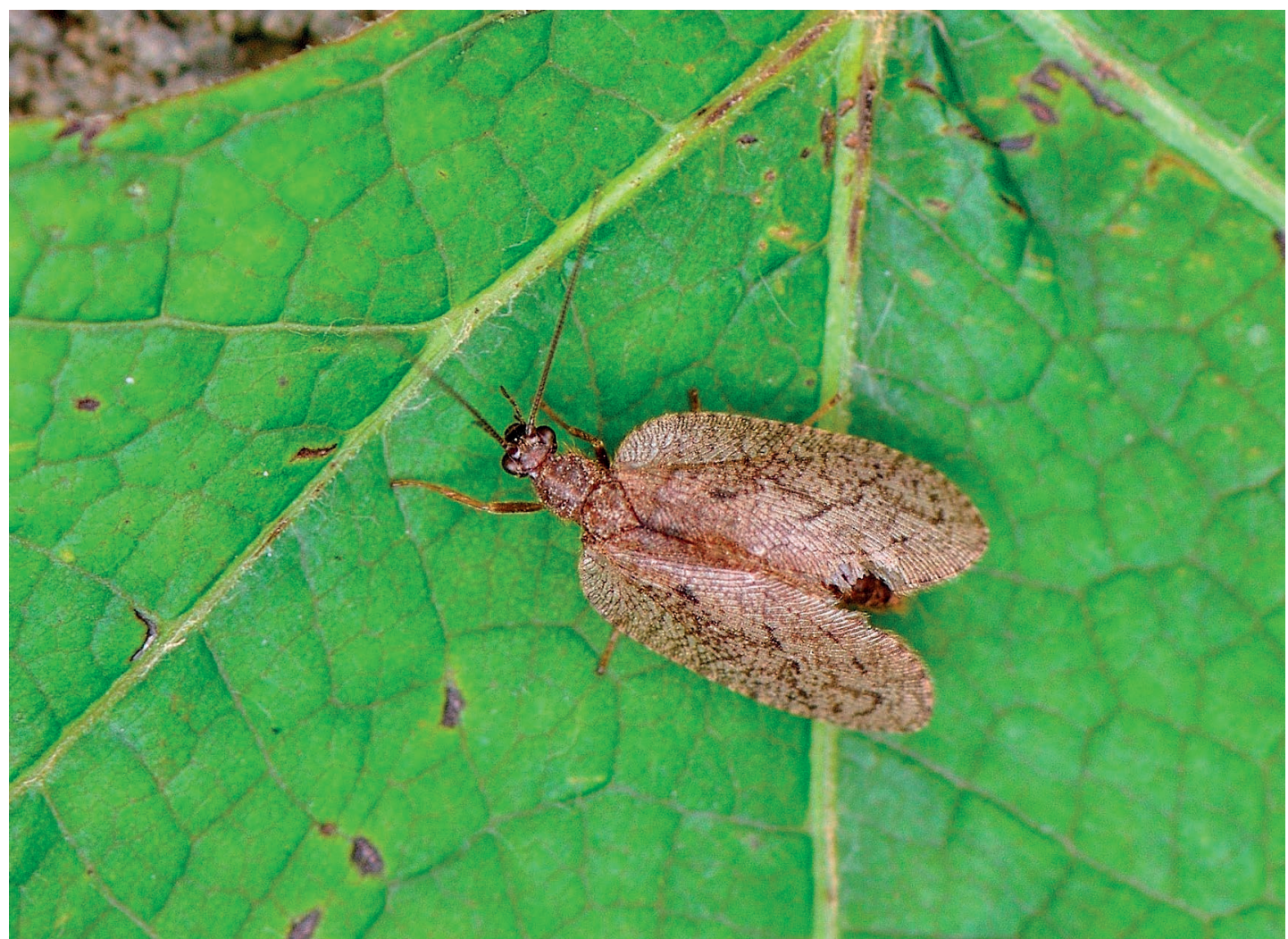

FIG. 2. - Drepanepteryx algida (Erichson in Middendorff, 1851), capturée pour la première fois en France dans le Parc national du Mercantour. Photographie : Jozef Šeršeň.

CATÉGORIE ZOOGÉOGRAPHiQUE. — Espèce polycentrique, centroeuropéenne et méditerranéenne.

Matériel eXaminé. — M09-SES1400T4-M2 (1 o7).

Distribution dans le Mercantour. — Saint-Dalmas-le-Selvage.

REMARQUE

Espèce nouvelle pour le Mercantour.

Famille MYrMELEONTIDAE Latreille, 1802

Genre Myrmeleon Linnaeus, 1767

Myrmeleon bore (Tjeder, 1941)

Grocus bore Tjeder, 1941: 73.

CATÉGORIE ZOOgÉOgRAPHIQUE. — Espèce sibérienne expansive.

Distribution dans le Mercantour. — Saint-Étienne-de-Tinée (Cocquempot \& Chambon 1990).

\section{REMARQUE}

Cette espèce à très large répartition n'est connue en France que de deux stations d'altitude, l'une pyrénéenne, l'autre alpine (Tillier 2010).
Genre Distoleon Banks, 1910

[Distoleon tetragrammicus (Fabricius, 1798)]

Myrmeleon tetragrammicus Fabricius, 1798: 205.

CATÉgorie ZOOGÉographiQue. — Espèce holoméditerranéenne expansive.

Distribution DANS LE MERCANTOUR. — [Barcelonnette] (Leraut 1992c).

Famille ASCALAPHIDAE Lefèbvre, 1842

Genre Libelloides Schäffer, 1763

\section{Libelloides coccajus}

(Denis \& Schiffermüller, 1775)

Papilio coccajus Denis \& Schiffermüller, 1775: 187.

CATÉGORIE ZOOGÉOGRAPHIQUE. — Espèce adriato-méditerranéenne expansive.

Distribution Dans Le Mercantour. - Saint-Dalmas-le-Selvage, Saint-Étienne-de-Tinée (Cocquempot \& Chambon 1990).

Libelloides lacteus (Brullé, 1832)

Ascalaphus lacteus Brullé, 1832: 278. 
CATÉGORIE ZOOGÉOGRAPHIQUE. — Espèce ponto-méditerranéenne.

Distribution dans le Mercantour. - Saint-Martin-Vésubie (Séméria \& Berland 1988).

\section{Libelloides longicornis}

(Linnaeus, 1764)

Hemerobius longicornis Linnaeus, 1764: 402.

CATÉGORIE ZOOGÉOGRAPHIQUE. — Espèce atlanto-méditerranéenne.

Distribution dans le Mercantour. - Saint-Martin-Vésubie (Luquet 1985); Bayasse (Leraut 1992c).

\section{DISCUSSION}

Au cours de ces trois années de piégeage, 36 espèces de Neuropterida ont été collectées. Les captures ont fourni un important matériel appartenant aux Hemerobiidae et aux Coniopterygidae. Par contre, les captures n’ayant été réalisées que par piégeage (Malaise et interception), les Chrysopidae et surtout les Myrmeleontidae et les Ascalaphidae n'ont été que peu ou pas collectées. Au total, six espèces de Raphidioptera et 30 espèces de Neuroptera ont été récoltées. Trois espèces ont été trouvées pour la première fois France: Drepanepteryx algida (Erichson in Middendorff, 1851), Helicoconis (Helicoconis) hirtinervis Tjeder, 1960 et Helicoconis (Fontenellea) hispanica Ohm, 1965. Neuf autres espèces sont nouvelles pour le Mercantour: Xanthostigma xanthostigma (Schummel, 1832), Coniopteryx (Coniopteryx) pygmea Enderlein, 1906, Coniopteryx (Coniopteryx) tineiformis Curtis, 1834, Parasemidalis fuscipennis (Reuter, 1894), Conwentzia psociformis (Curtis, 1834), Hemerobius (Hemerobius) schedli Hölzel, 1970, Sympherobius (Sympherobius) elegans (Stephens, 1836), Megalomus hirtus (Linnaeus, 1761) et Micromus (Micromus) variegatus (Fabricius, 1793). En l'état actuel des connaissances, la liste des Neuropterida du Parc national du Mercantour s'établit ainsi à six Raphidioptera et 46 Neuroptera. À cette liste s'ajoutent quatre espèces capturées dans des stations situées sur des communes limitrophes au Parc national du Mercantour et sans aucun doute présentes à l'intérieur des limites du Parc.

La poursuite d'inventaires tels que celui réalisé dans le cadre de l'ATBI devrait permettre d'enrichir encore les connaissances sur la faune des Neuropterida de cette région.

\section{Remerciements}

L'Inventaire biologique généralisé (IBG) Mercantour/Alpi Marittime a été mis en place par le projet the European Distributed Institute of Taxonomy (EDIT) (2006-2011). Nous remercions M.-F. Leccia (Parc national du Mercantour) et M. De Biaggi (Parco Alpi Marittime) pour leur coordination de l'IBG ainsi que pour avoir fourni l'ensemble des autorisations nécessaires aux collectes de terrain.

Les spécimens étudiés dans cet article ont été collectés dans le cadre du module Invertébrés terrestres du Muséum national
d'Histoire naturelle; les pièges de malaise ont été récoltés par J. Molto, M. Torjman et E. Minssieux (Parc national du Mercantour) et trié à la famille par M. Czyrnek et T. Théry (MNHN). J'adresse également mes vifs remerciements à Louis Deharveng, Christophe Daugeron et Claire Villemant (UMR7205, ISYEB) pour m'avoir permis d'examiner les Neuropterida collectés dans ce cadre. Enfin, je tiens particulièrement à remercier Jozef Šeršeň, qui a très généreusement mis à disposition l'une de ses excellentes photographies, afin d'illustrer cette publication. Je suis également reconnaissant envers Patrice Leraut, ainsi qu'envers plusieurs relecteurs anonymes, pour leurs remarques constructives.

\section{RÉFÉRENCES}

Albarda H. 1891. - Révision des Rhaphidides. Tijdschrift voor Entomologie 34: 65-184.

AspöсK H. \& AsPÖсK U. 1966. — Studien an europäischen und kleinasiatischen Arten des Genus Raphidia L. (Insecta, Raphidiodea). Mitteilungen der Schweizerischen Entomologischen Gesellschaft 39: 33-48.

AsPÖCK H. \& AsPÖCK U. 1976. — Die Auflösung des Raphidia notata - Komplexes (Neur. Raphidioptera, Raphidiidae). Zeitschrift der Arbeitsgemeinschaft Österreichischer Entomologen 27: 57-70.

AsPÖCK H., AsPÖCK U. \& RAUSCH H. 1974a. — Bestimmungsschlüssel der Larven der Raphidiopteren Mitteleuropas (Insecta, Neuropteroidea). Zeitschrift für Angewandte Entomologie 61: 45-62.

AsPÖCK H., AsPÖCK U. \& HÖLZEL H. 1980. - Die Neuropteren Europas. Eine zusammenfassende Darstellung der Systematik, Ökologie und Chorologie der Neuropteroidea (Megaloptera, Raphidioptera, Planipennia) Europas. Goecke \& Evers, Krefeld, F. R. G., 2 vol., p. 495,355 .

Aspöck H., Aspöck U. \& Rausch H. 1991. - Die Raphidiopteren der Erde. Eine monographische Darstellung der Systematik, Taxonomie, Biologie, Okologie und Chorologie der rezenten Raphidiopteren der Erde, mit einer zusammenfassenden Übersicht der fossilen Raphidiopteren (Insecta: Neuropteroidea). Goecke \& Evers: Krefeld, 2 vols, p. 730, 550.

AsPÖCK H., HÖlZEl H. \& AsPÖCK U. 2001. - Kommentierter Katalog der Neuropterida (Insecta: Raphidioptera, Megaloptera, Neuroptera) der Westpaläarktis. Denisia 2: 1-606.

AsPÖCK H., RAUSCH H. \& AsPÖCK U. 1974b. - Untersuchungen über die Ökologie der Raphidiopteren Mitteleuropas (Insecta, Neuropteroidea). Zeitschrift für Angewandte Entomologie 76: 1-30.

BRAUER F. 1851. - Beschreibung und Beobachtung der österreichischen Arten der Gattung Chrysopa. Naturwissenschaftliche Abhandlungen, gesammelt und durch subscription herausgegeben von Wilhelm Haidinger 4 (4): 1-12.

Brauer F. 1876. - Die Neuropteren Europas und insbesondere Oesterreichs mit Rücksicht auf ihre geographische Verbreitung, in Festschrift zur Feier des fünfundzwanzigjährigen Bestehen Kaiserlich-Königlichen Zoologisch-Botanischen Gesellschaft, Wien, p. 263-300.

BRullé G. A. 1832. — Expédition scientifique de Morée. Tome 3, Partie 1 (Zoologie), Section 2 (Des animaux articulés). Levrault, Paris.

BuRMEISTER H. C. C. 1839. - Handbuch der Entomologie. Zweiter Band - Volume II. Kaukerfe. Gymnognatha. Theod. Chr. Friedr. Enslin, Berlin. [i]-xii + 757-1050 p.

Canard M., Cloupeau R. \& Leraut P. 1998. — Les Chrysopes du genre Nineta Navás, 1912, en France (Neuroptera, Chrysopidae). Bulletin de la Société entomologique de France 103 (4): 327-336.

Canard M., Giacomino M., Thierry D., Tillier P. \& VillenaveChasset J. 2011. - Deuxième complément à la cartographie 
des Chrysopes de France (Neuroptera: Chrysopidae). Revue de l'Association roussillonnaise d'Entomologie 20 (2): 45-52.

COCQuempot C. \& ChAmbon J.-P. 1990. — Contribution à l'étude de la faune entomologique du Parc national du Mercantour et ses environs immédiats. (Neuroptera, Raphidioptera, Planipennia, Orthoptera, Dermaptera, Lepidoptera, Coleoptera, Hymenoptera, Hemiptera, Homoptera, Diptera). Biocosme mésogéen 7 (3-4): 133-150.

Costa A. 1855. - Famiglia de' Rafidiidei - Rhaphidiidea, in Costa A., Fauna del Regno di Napoli ossia enumerazione di tutti gli animali che abitano le diverse regioni di questo Regno e le acque che le bagnano e descrizione dénuovi o poco esattamente conosciuti con figure ricavate daoriginali viventi e dipinte al naturale. Nevrotteri.- Stamperia di Antonio Cons, Napoli. 83 p.,7 pls.

CURTIS J. 1834. - British entomology; being illustrations and descriptions of the genera of insects found in Great Britain and Ireland: containing coloured figures from nature of the most rare and beautiful species, and in many instances of the plants upon which they are found. London. 16 vols.

Deharveng L., Bedos A., Daugeron C., Villemant C. \& Judson M. L. I. 2015. - Organization, usefulness and limitations of an ATBI (All Taxa Biodiversity Inventory): the inventory of terrestrial invertebrates in the Mercantour National Park, in DAUGERON C., Deharveng L., Isaia M., Villemant C. \& Judson M. (eds), Mercantour/Alpi Marittime All Taxa Biodiversity Inventory. Zoosystema 37 (1): 9-30. http://dx.doi.org/10.5252/z2015n1a1

DENIS M. \& SCHIFFERMÜLlER I. 1775. - Ankündung eines systematischen werks von der schmetterlingen der Wienergegend. A. Bernardi, Wien, $322 \mathrm{p}$.

Enderlein G. 1906. - Monographie der Coniopterygiden. Zoologische Jahrbücher (Abteilung für Systematik, Geographie und Biologie) 23: 173-242.

ERICHSON W. F. 1851. — Nevroptera in MÉNÉTRIÉs E., Die Insecten in MidDENDORF A. T. VON (ed.), Reise in den äussersten norden und osten Sibiriens während der jahre 1843 und 1844 mit allerhöchster genehmigung aufveranstaltung der Kaiserlichen Akademie der Wissenschaften zu St. Petersburg ausgeführt und in verbindung mit vielen gelehrten herausgegeben von Dr. A. Th. v. Middendorf. Bd. 2, Theil 1. Kaiserlichen Akademie der Wissenschaften, St. Petersburg, p. 68-69.

FABRICIUS J. C. 1793. - Entomologia systematica emendata et aucta secundum classes, ordines, genera, species adjectis synonimis, locis observationibus, descriptionibus. Tome 2. C. G. Proft, Hafniae. 519 p.

FABRICIUS J. C. 1798. — Supplementum entomologiae systematicae. Hafniae. 572 p.

Fraser F. C. 1942. - A new species of Kimminsia (Neur. Hemerobiidae), with historical figures and notes by R. McLachlan and K. J. Morton. Entomologist's Monthly Magazine 78: 80-86.

GÜSTEN R. 1998. - 332. Raphidia ligurica Albarda, 1891 (Raphidioptera Raphidiidae). Bollettino della Società Entomologica Italiana 130 (1): 81.

Haring E., AsPÖCK H., BARTEl D. \& AsPÖCK U. 2011. — Molecular phylogeny of the Raphidiidae (Raphidioptera). Systematic Entomology 36: 16-30.

Hölzel H. 1970. - Ein neuer Hemerobius aus Mitteleuropa (Planipennia, Hemerobiidae). Nachrichtenblatt der Bayerischen Entomologen 18: 104-106.

Killington F. J. 1933. - A new species of Boriomyia (Neur., Hemerobiidae) from France. Entomologist's Monthly Magazine 69: 57-59.

Killington F. J. 1935. - Chrysopa albolineata nom. nov. for Chrysopa tenella Schneid. (Neur.). Journal of the Society for British Entomology 1: 87.

LACroIX J. L. 1912. - Faune névroptérique de l'Algérie et de la Tunisie. I. Deux espèces nouvelles. Insecta, Rennes 2: 202-206.

LERAUT P. 1988. - Cunctochrysa bellifontensis n. sp.: une espèce de chrysope nouvelle pour la science découverte en forêt de Fontainebleau (Neur. Chrysopidae). Bulletin de l'Association des
Naturalistes de la Vallée du Loing et du Massif de Fontainebleau 64: 230-235.

LERAUT P. 1989. - Étude de la variation subspécifique de Metachrysopa pallens (Rambur, 1838) n. comb. [Neuroptera, Chrysopidae]. Revue Française d'Entomologie (N.S.) 11: 105-108.

LERAUT P. 1991a. - Hemerobius micans (Olivier) sur des pins au col de la Cayolle vers 2300 m (Alpes-Maritimes) (Neur. Hemerobiidae). Entomologica Gallica 2 (3): 153.

Leraut P. 1991b. - Contribution à l'étude des hémérobes. 3. Hemerobius ballaudi sp. n. en France (Neur. Hemerobiidae). Entomologica Gallica 2 (4): 189-190.

LERAUT P. 1991c. - Contribution à l'étude des hémérobes. 4. Kimminsia cunctatus $(\mathrm{Ohm})$ et Hemerobius fenestratus Tjeder en France; nouvelles données sur la distribution de Micromus lanosus (Zeleny) (Neur. Hemerobiidae). Entomologica Gallica 2 (4): 215-216.

LERAUT P. 1992a. — Quelques névroptères nouveaux pour la France, dont une espèce et une sous-espèce nouvelles pour la science (Neur.). Entomologica Gallica 3 (1): 7-9.

LERAUT P. 1992b. - Chrysopa gibeauxi (Leraut), stat. rev. une espèce distincte (Neur. Chrysopidae). Entomologica Gallica 3 (1): 24-26.

Leraut P. 1992c. - Névroptères des Alpes centrales françaises (Neur.). Entomologica Gallica 3 (2): 59-65.

LINNAEUS C. 1758. - Systema natura per regna tria naturae secundum classes, ordines, genera, species, cum characteribus, differentiis, synonymis, locis. Editio decima, reformata [10e édition]. Tome I. Salvii, Holmiae, 824 p.

LINNAEUS C. 1761. - Fauna Svecica, sistens animalia Sveciae regni: Mammalia, Aves, Amphibia, Pices, Insecta, Vermes distributa per classes \& ordines, genera \& species, cum differentiis specierum, synonymis auctorum, nominibus natalium, descriptionibus insectorum. Editio altera, auctior [ $=2 \mathrm{e}$ édition]. Laurentii Salvii, Stockholmiae. 578 p.

LINNAEUS C. 1764. - Museum ludovicae ulricae reginae svecorum, gothorum, vandalorumque. In quo animalia rariora, exotica, imprimis insecta \& Conchilia describuntur \& determinantur prodomi instar editum. Laur. Salvii, Holmiae, 720 p.

LINNAEUS C. 1767. - Systema natura per regna tria naturae secundum classes, ordines, genera, species, cum characteribus, differentiis, synonymis, locis. Editio duodecima reformata [ $=12 \mathrm{e}$ édition]. Tome I.

LUQUET G.-C. 1985. — Quelques précisions sur la répartition de plusieurs névroptères français (Neur. Ascalaphidae, Myrmeleonidae). Entomologica Gallica 1 (4): 314-315.

MCLACHLAN R. 1868. - A monograph of the British NeuropteraPlanipennia. Transactions of the [Royal] Entomological Society of London 16: 145-224.

Monserrat V. J. \& Papenberg D. 2010. - Revison of the genus Phaeostigma Navás, 1909 from the Iberian Peninsula (Insecta, Raphidioptera). Graellsia 66 (1): 47-77.

NAVÁS L. 1925. - Comunicaciones entomológicas. 7. Neurópteros del Museo de Berlín. Revista de la Academia de Ciencias Exactas Fisico-Quimicas y Naturales de ZaragoZa 9 (1): 20- 34.

Oнм P. 1965. - Beiträge zur Kenntnis der Gattung Helicoconis Enderlein 1905 (Neuroptera, Coniopterygidae) nebst Diagnose zweier neuer Arten aus dem schweizerischen nationalpark. Ergebnisse der Wissenschaftlichen Untersuchungen des Schweizerischen Nationalparks 10: 169-207.

Olivier G. A. 1792. — Encyclopedie méthodique. Histoire naturelle. Vol. 7 (Insectes), Paris.

Rambur P. 1842. - Histoire naturelle des Insectes, Névroptères. Librairie encyclopédique de Roret. Fain et Thunot, Paris, 534 p.

Rausch H., AsPÖCK H. \& AsPÖCK U. 2004. - Calabroraphidia renate n. gen., n. sp. - eine neue Spezies und ein neues Genus der Familie Raphidiidae aus Süditalien (Neuropterida, Raphidioptera). Entomologische Nachrichten und Berichte 48 (3/4): 159-165.

Reuter O. M. 1894. - Neuroptera Fennica. Förteckning och Beskrifning öfver Finlands Neuropterer. Acta Societatis pro Fauna et Flora Fennica 9 (8): 1-36.

RufFo S. \& STOCH F. (eds) 2006. - Checklist and Distribution of the Italian Fauna. Memorie del Museo Civico di Storia Natu- 
rale di Verona. 2. Serie. Sezione Scienze della Vita 17 (données sur CD-Rom).

SCHNEIDER W. G. 1846. - Ueber die schlesischen Hemorobius[sic]-Arten. Uebersicht der Arbeiten und Veränderung der Schlesischen Gesellschaft für Vaterländische Cultur 1846: 100-102.

SCHNEIDER W. G. 1851. - Symbolae ad monographiam generi Chrysopae, Leach. Sexaginta picturarum tabulis, in lapide acu delineatis, quarum quinquaginta quatuor coloribus impressae sunt, illustratae. Editio major. Ferdinandum Hirt, Vratislaviae, 178 p.

SCHummel T. E. 1832. - Versuch einer genauen Beschreibung der in Schlesien einheimischen Arten der Gattung Raphidia, Linn. Beiträge zur entomologie besonders in bezug aufSchlesien, herausgegeben von T. E. Schummel. Eduard Pelz, Breslau. $\mathrm{N}^{\circ} 1,16$ p.

SÉméRIA Y. \& BERLAND L. 1988. — Atlas des Névroptères de France et d'Europe. Mégaloptères - Raphidioptères - Névroptères Planipennes - Mécoptères. Société nouvelle des éditions Boubée, Paris, 190 p.

SCOPOLI J. A. 1763. - Entomologia Carniolica, exhibens insecta Carnioliae indigena et distributa in ordines, genera, species, varietates, methodo Linneana. Ioannis Thomae Trattner, Vindobonae. $418 \mathrm{p}$.

STEPHENS J. F. 1836. - Illustrations of British entomology; or, a synopsis of indigenous insects: containing their generic and specific distinctions; with an account of their metamorphoses, times of appearance, localities, food, economy, as far as practicable. Mandibulata Vol. 6. Baldwin and Cradock, London. 240 p.

STEIN J. P. E. F. 1863. — Beitrag zur Neuropteren-fauna Griechenlands (mit Berücksichtigung dalmatinischer Arten). Berliner Entomologische Zeitschrift 7: 411-422.

Tillier P. 2009. - Liste des Megaloptera de France (version du 28-XII-2009). http://www.insecte.org/forum/viewtopic. php?f $=33 \& \mathrm{t}=59629$

Tillier P. 2010. - Capture en Corse de Neuroleon microstenus (McLachlan 1898), nouvelle espèce pour la France, et nouvelles données sur des fourmilions rares ou peu connus en France (Neuroptera Myrmeleontidae). L'Entomologiste 66 (2): 73-80.

Tillier P. 2012a. - Liste des Raphidioptera de France (version du 13-VI-2012). http://www.insecte.org/forum/viewtopic. php?f $=33 \& \mathrm{t}=59630$

TILlier P. 2012b. - Drepanepteryx algida (Erichson in Middendorff, 1851) dans le Mercantour, espèce nouvelle pour la faune de France et liste actualisée des Hemerobiidae de France (Neuroptera). Bulletin de la Société entomologique de France 117 (4): 457-459.
Tillier P. 2012c. - Présence de Hemerobius (Hemerobius) schedli (Hölzel, 1970) dans les Alpes françaises (Neuroptera Hemerobiidae). L'Entomologiste 68 (4): 253-254.

Tillier P. 2013a. - Liste des Neuroptera de France (version du 25-VI-2013). http://www.insecte.org/forum/viewtopic. php?f $=338 \mathrm{t}=59631$

TILLIER P. 2013b. - Nouvelles captures de Raphidia (Raphidia) ligurica Albarda, 1891 et de Phaeostigma (Phaeostigma) italogallica (H. Aspöck \& U. Aspöck, 1976) dans le Mercantour (Raphidioptera Raphidiidae). L'Entomologiste 69 (2): 121-123.

TilLIER P. 2013c. - Deux espèces du genre Helicoconis Enderlein, 1905, nouvelles pour la France et liste actualisée des Coniopterygidae de France (Neuroptera). Bulletin de la Société entomologique de France 118 (2): 141-144.

Tillier P., Thierry D., Dobosz R. \& Canard M. 2014. - Chrysopa gibeauxi (Leraut, 1989): reinstatement as valid species and remarks on its distribution (Neuropterida, Chrysopidae). Bulletin de la Société entomologique de France 119 (4): 521-528.

Tjeder B. 1932. - Preliminary notes on Hemerobius limbatellus of authors. Entomologisk Tidskrift 53: 193-195.

TJeder B. 1941. - A new species of Myrmeleontidae from Scandinavia. Preliminary description. Opuscula Entomologica 6: 73-74

TJEDER B. 1960. - A new subapterous coniopterygid from Denmark (Neuroptera). Entomologiske Meddelelser 29: 312-319.

TJEDER B. 1963. - Comments on Hemerobius algidus Erichson, with notes on the genus Drepanopteryx [sic] (Neur. Hemerobiidae). Opuscula Entomologica 28: 171-180.

Villemant C., Daugeron C., Gargominy O., Isaia M., DeharVENG L. \& JUDSON M. L. I. 2015. — The Mercantour/Alp Marittime All Taxa Biodiversity Inventory (ATBI): achievements and prospects, in DAUgeron C., Deharveng L., IsAiA M., VILLEMANT C. \& JUDSON M. (eds), Mercantour/Alpi Marittime All Taxa Biodiversity Inventory. Zoosystema 37 (4): 667-679. http:// dx.doi.org/10.5252/z2015n4a10

WALKER F. 1853. - List of the specimens of neuropterous insects in the collection of the British Museum. Part II - Sialides-Nemopterides. British Museum, London : 193-476.

WaLLENGREN H. D. J. 1863. — Bidrag till kännedomen af Sveriges Neuroptera. Öfversigt af Kongl. Vetenskaps-Akademiens Forhändlingar 20: 15-26.

ZELENÝ J. 1962. - Stenomicromus lanosus n. sp., a new species of Neuroptera from Czechoslovakia (Neuroptera, Hemerobiidae). Casopis Ceskoslovenské Spolecnosti Entomologické 59: 246-249. 